\title{
Evaluation of Laboratory Performance Through Interlaboratory Comparison
}

\author{
SANJAY YADAV and A.K. BANDYOPADHYAY \\ National Physical Laboratory, New Delhi - 110 012, India \\ e-mail: syadav@mail.nplindia.ernet.in
}

[Received: 16.05.2009; Accepted: 16.06.2009]

\begin{abstract}
This paper reports the performance of the calibration results obtained by 21 laboratories using digital pressure calibrator as an artifact in the pressure range 7-70 MPa. National Physical Laboratory (NPLI), New Delhi has coordinated this programme and also acted as a reference laboratory. The program started in May, 2006 and completed during May, 2008. The comparison was carried out at 10 equally spaced pressure points i.e. 7, 14, 21,28, 35, 42, 49, 56, 63 and $70 \mathrm{MPa}$ throughout the entire pressure range of $7-70 \mathrm{MPa}$. The calibration results thus obtained were analyzed as per ISO/ IEC GUM document. The $92.7 \%$ measurement results are found in agreement with the results of NPLI. The relative deviations between laboratories values and reference values are well within the 0.05 \% for 123 measurement points, $0.1 \%$ for 162 measurement points and $0.25 \%$ for 177 measurement points. The difference of the laboratories values with reference values are found almost well within the uncertainty band of the reference values at $68.0 \%$ measurement results, within their reported expanded uncertainty band at $81.5 \%$ measurement results and within the combined expanded measurement uncertainty band at $92.7 \%$ measurement results. Overall, the results are considered to be reasonably good being the first proficiency testing for most of the participating laboratories.
\end{abstract}

\section{Introduction}

The last few decades have witnessed the growing need of the industrial utilization of pressure metrology day by day. The control, monitor and measurement of pressure are not only important to increase the productivity but also play a vital role from the safety point of view. As we move in to the new millennium, the globalization and international competitiveness, the international trade and commerce will continue to significantly impact our economy. As trade barriers are lifted, our economy, in particular manufacturers must be prepared to comply with international standards, including the assessment of competency in measurements.

(c) Metrology Society of India, All rights reserved.
In order to establish the international / national compatibility, uniformity and affirmation of measurement results, considerable efforts are being made globally so that the measurements made in one location in the world are equivalent / compatible in other locations on the same or related products. Such tasks are achieved by organizing international comparisons and proficiency testing by inter-laboratory comparisons of the measurement results carried out on the same artifact. The National Measurement Institutes (NMIs) provide traceability to industries and other users in terms of calibration.

The Mutual Recognition Arrangement (MRA) also stipulates the requirement for measurement 
laboratory to participate in the proficiency testing and establish the technical competence. To meet the requirements of MRA [4], ISO 17025 [5] and APLAC MR001 [6], the National Accreditation Board for Testing \& Calibration Laboratories (NABL) has conducted several Proficiency Testing (PT) experiments in pressure metrology in the pressure range 5-70 $\mathrm{MPa}$ amongst the NABL accredited Indian pressure calibration laboratories in conformity with ISO/IEC Guide 43 [7] through the National Metrology Institute (NMI) of India i.e. National Physical Laboratory (NPLI), New Delhi which acts as a Reference laboratory.

In a series of 7 PTs organized, the 1st and 4th PTs, designated as NABL-Pressure-PT001 and NABL-Pressure-PT004 were organised / being organised for 7 and 10 laboratories, respectively having measurement capabilities better than $0.05 \%$ of full scale pressure using dead weight tester as an artifact [8-9]. The $2^{\text {nd }}$ PT i.e. NABL-Pressure-PT002 was conducted for another 7 laboratories, having measurement capabilities coarse than $0.05 \%$ and better than $0.25 \%$ of full scale pressure using digital pressure calibrator [10-11]. The $3^{\text {rd }}$ and $6^{\text {th }}$ PTs i.e. NABL-Pressure-PT003 and NABL-Pressure-PT006, included 11 and 17 laboratories, respectively having measurement capabilities $0.25 \%$ or coarse than $0.25 \%$ of full scale pressure using pressure dial gauge as an artifact [12-13]. Similarly, another PT experiment i.e. NABL-Pressure-PT007 was carried out for 14 laboratories having measurement capabilities $0.25 \%$ or coarse than $0.25 \%$ of full scale pressure using pressure dial gauge as an artifact in the pressure range 6 - 60 $\mathrm{MPa}[14]$.

The present PT experiment, designated as NABLPressure-PT005, is recently completed during May 2008. This PT programme is designed and organized in the hydraulic pressure region covering pressure range $7-70 \mathrm{MPa}$ (70 to 700 bar) using the digital pressure calibrator as an artifact. The 21 NABL accredited pressure calibration laboratories, having measurement capabilities coarser than $0.05 \%$ and better than $0.25 \%$ of full scale pressure have been covered in this PT. The report summarizes the results of measurement of pressure at equally spaced pressure points. The circulation of the artifact started in July 2006 and completed in April 2008 in a record time.

\section{Methodology}

The PT programme is designed as per guidelines stipulated in ISO/IEC 17025 [5], ISO/IEC Guide 43 [7] and NABL-162 [15]. The artifact used for the measurements is a high precision Digital Pressure Calibrator, Serial No. H540/101, make-DHBudenberg, UK. Selection of the measurement points is an important aspect of the proficiency testing programme. The entire pressure range of 7 to $70 \mathrm{MPa}$ was divided into 10 equally spaced measurement points of 7, 14, 21, 28, 35, 42, 49, 56, 63 and $70 \mathrm{MPa}$. The programme was running smoothly and almost all the participants performed their measurements well in time. Generally, all the participants were advised to complete the measurements in two weeks time and dispatch the artifact to next participant within next one week. However, one week time for measurements and one week time for dispatch was given for the participants when artifact was moved within the same city. The whole circulation programme was completed in two loops. The movement of the artifact was smoothly monitored. After smooth completion of the programme of the first loop, the artifact reached NPLI, New Delhi during April, 2007. It was recalibrated at NPLI. New Delhi and then dispatched for the circulation of the second loop. There was no technical problem, fault, snag or difficulty reported by any of the participants. Schematic diagram of the movement of the artifact is depicted in Fig. 1.

\section{Characterization of the Artifact and Assigning Reference Values}

The characterization of the artifact was performed by direct comparison method [1-4] against the national hydraulic secondary pressure standard, designated as NPL200MPA, first at the start of the programme during June, 2006, second in the middle during March-April, 2007 and finally at the end of programme during April, 2008. The traceability of the NPL200MPA is established by cross-floating it against national secondary pressure standards [16-19] and its measurement uncertainty is estimated as $68 \times 10^{-6} \times p$ at a coverage factor $k=1$. NPL200MPA has also been used as NPL standard to calibrate the transfer standards for the international key comparison i.e. APMP-SIM. M.P. K-7 [20]. In this comparison, our results agreed well within $7.7 \times 10^{-6}$ with NIST, USA and were also found well within 


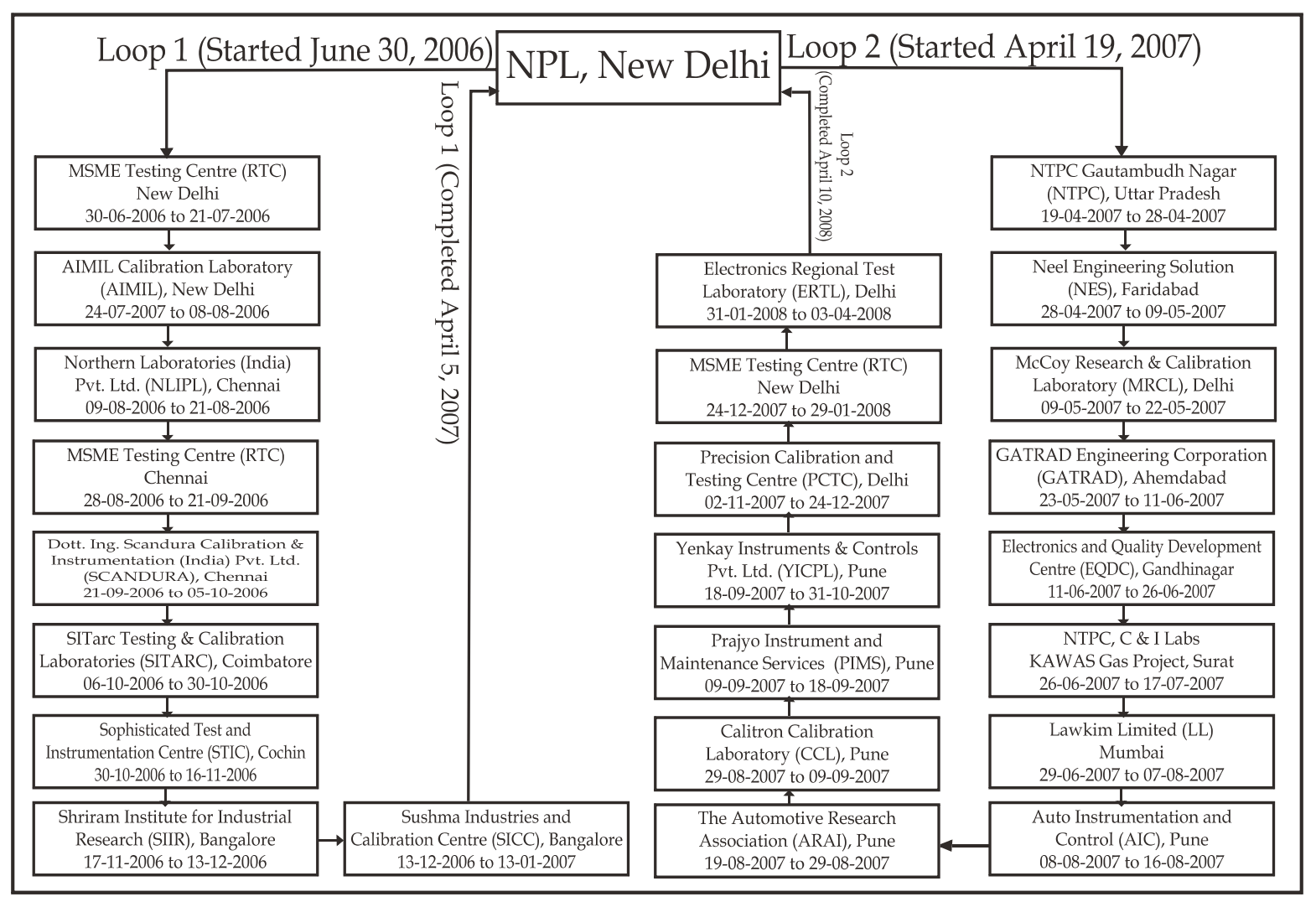

Fig. 1. Circulation and movement of the artifact during comparison

our claimed measurement standard uncertainty of $40 \times 10^{-6}$. Measurement were performed at 10 pressure points i.e. 7, 14, 21, 28, 35, 42, 49, 56, 63 and $70 \mathrm{MPa}$ and observations were repeated six times, thrice in increasing order and thrice in decreasing order, for each pressure point and the values of measured pressure, their repeatability and expanded uncertainty were computed using method described and computer software developed for this purpose [21-23].

The values of measured pressures $p_{1} p_{2}$ and $p_{3}$, for three successive calibrations, during June, 2006, March-April, 2007 and April, 2008 are shown in Table 1 along with the details of the uncertainty computation. The arithmetic mean of these pressure values $\left(p_{1}, p_{2}\right.$ and $\left.p_{3}\right)$ are the reference values of the pressure measured $(p)$ for individual measurement point throughout the entire pressure scale. In order to study the behaviour and stability of the artifact, the calibration factor $\left(C_{p}\right)$ is determined as follows;
$C_{f}=\frac{p_{g}}{p_{S}}$

where, $p_{g}$ is the reading of the artifact and $p_{S}$ is corresponding pressure measured by the standard during calibration. The behavior of the artifact was found identical in all the three calibrations. It is clearly evident from Fig. 2(a) that during all the three calibrations, the artifact behaved almost in a similar fashion. Although, the behaviour of the artifact was slightly different during March 2007 but the values of calibration factor were found much closer to the unity which indicates a close agreement between gauge reading and the standard pressure. The relative deviations of the measured pressures $p_{1}, p_{2}$ and $p_{3}$ from the reference values, $p$ are found well within $\pm 0.033 \%$ of the reading [Fig. 2(b)] and $\pm 0.01 \%$ of full scale [Fig. 2(c)]. The deviations are well within the manufacturer specifications of $0.05 \%$ of span (full scale), the maximum deviation of 0.033 $\%$ of reading is taken into consideration to estimate 
Sanjay Yadav and A.K. Bandyopadhyay

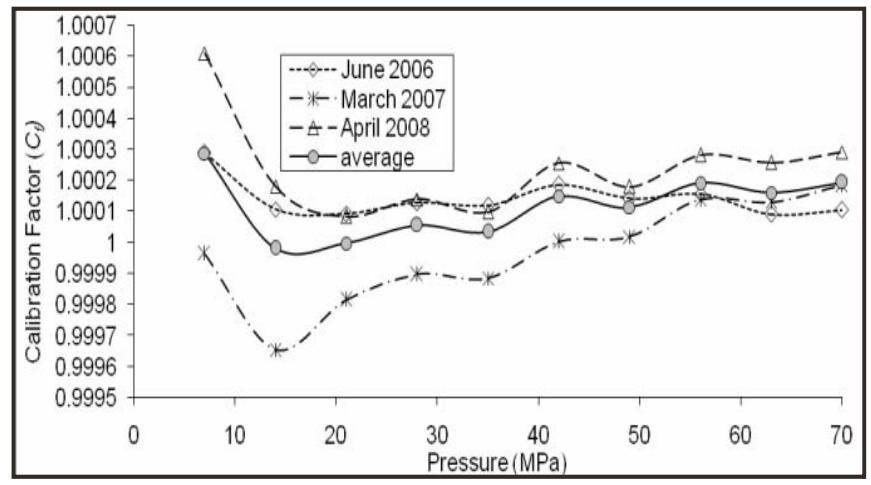

Fig. 2(a). The Calibration factor $\left(C_{\text {C }}\right.$ ) and its average values plotted as a function of applied pressure $p$ for all the three successive calibrations of the artifact

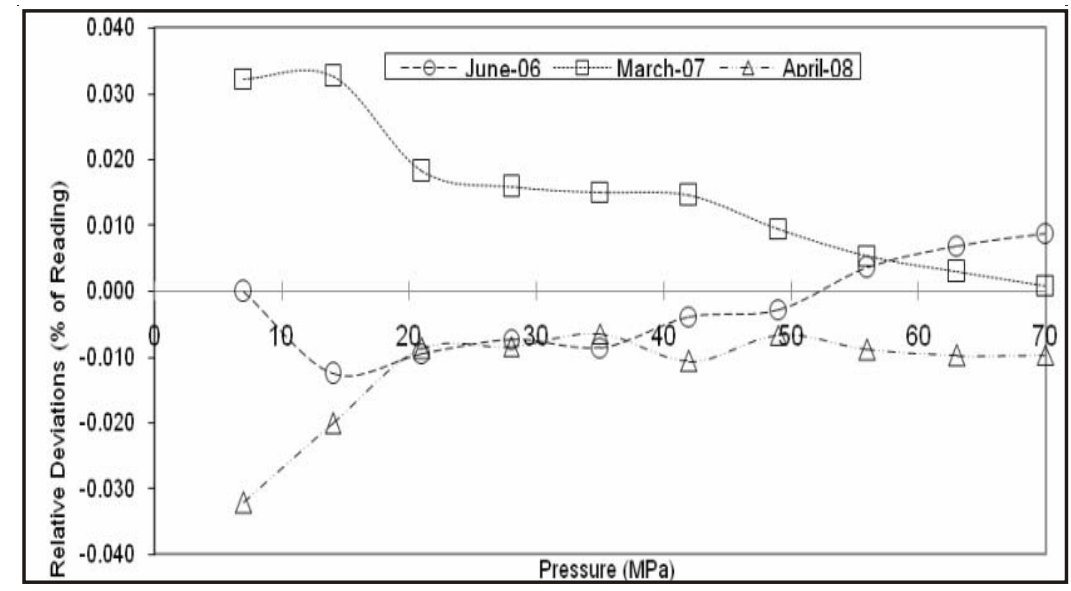

Fig. 2(b). Relative deviations ( $\%$ of reading) of the measured pressures $p_{1^{\prime}} p_{2}$ and $p_{3}$ from the reference values $p$ for all the three successive calibrations

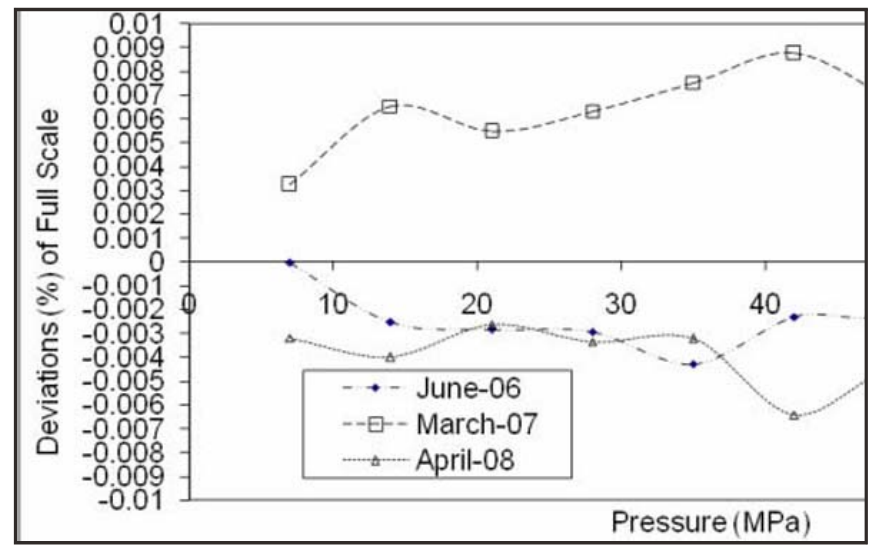

Fig. 2(c). Relative deviations (\% of full scale pressure) of the measured pressures $p_{1}, p_{2}$ and $p_{3}$ from the reference values $p$ for all the three successive calibrations 
Evaluation of Laboratory Performance through Interlaboratory Comparison

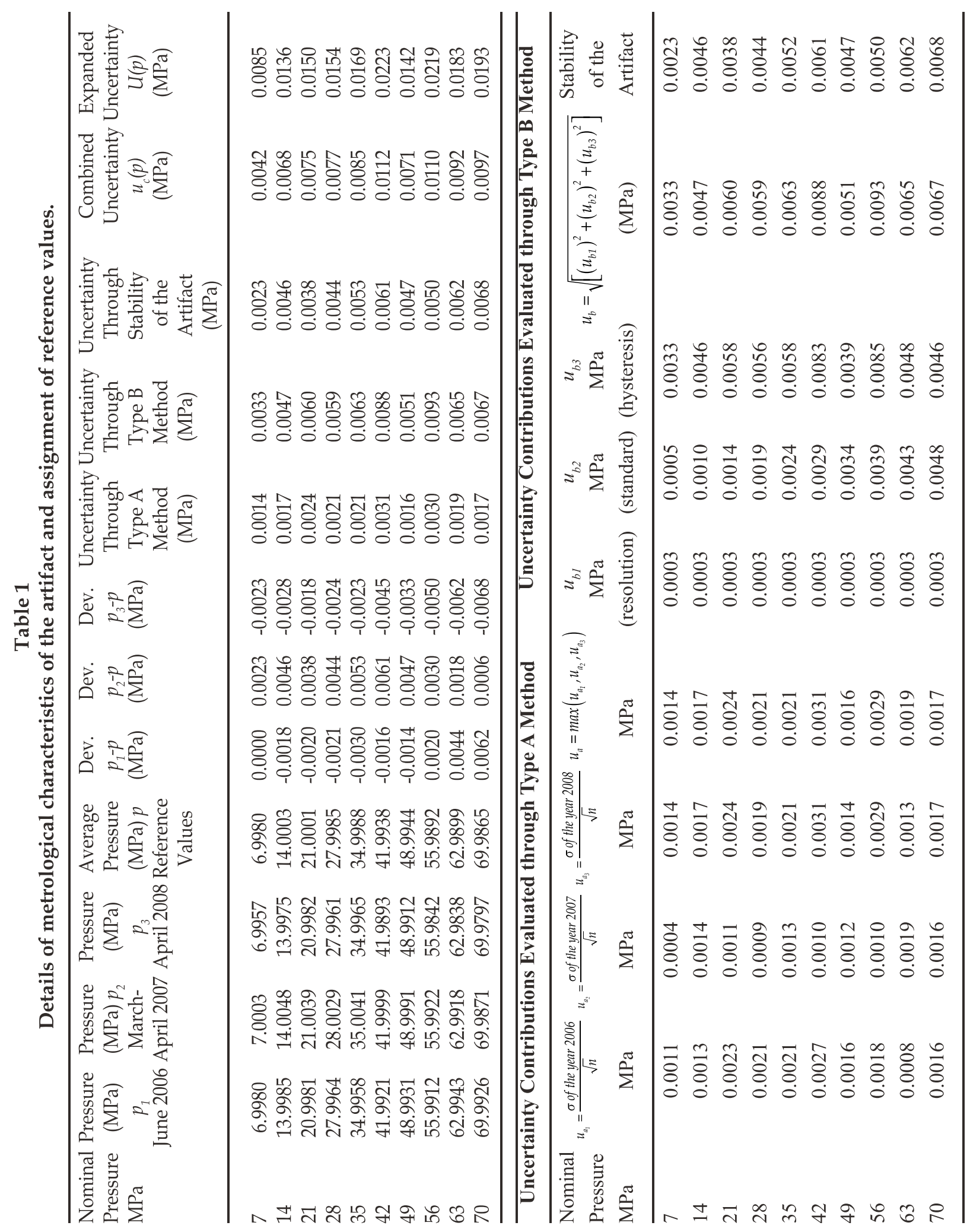




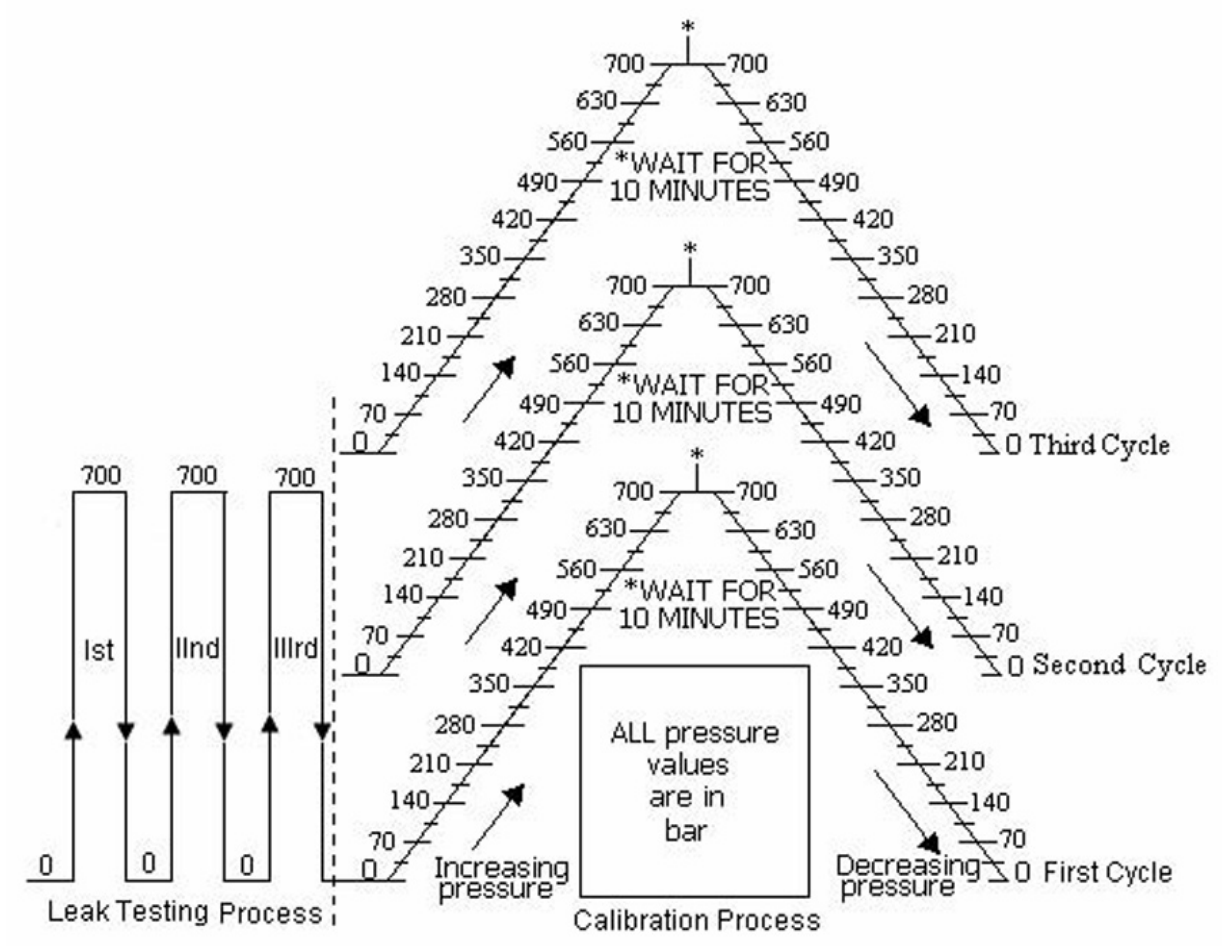

Fig. 3. Sequence of measurements taken

the expanded uncertainty $U(p)=(0.011+0.00015 p)$ $\mathrm{MPa}$. This concludes that the artifact remained stable during the whole PT programme within its estimated measurement uncertainty.

\section{Calibration Procedure}

A total of 21 laboratories participated in the program. Each participating laboratory was assigned a random code number while the reference laboratory, NPLI, was assigned '1.' The calibration procedure was identical to method used in NABLPressure - PT004 [10-11]. A layout diagram showing the sequence of measurements performed by each participant is shown in Fig. 3. After performing leak testing, zero setting, selection of datum level and generating pressure up to full scale, the participants were advised to wait for at least 10 minutes, before repeating the observations in the decreasing order of pressure till the pressure reaches to zero and then record the total 22 observations, 11 each in increasing and decreasing order of pressures for one cycle. The measurements were then repeated for at least 3 pressure cycles to make the total number of 66 observations.
They were requested to correct the values of the measured pressure for $23^{\circ} \mathrm{C}$ using thermal expansion coefficient of the piston - cylinder assembly (if dead weight tester is used as standard) or elastic element (if pressure dial gauge or digital calibrator is used as standard) using standard equations. They were also requested to evaluate the uncertainty associated with pressure measurements as per ISO Guide to the Expression of Uncertainty in Measurement/ NABL Document 141 on uncertainty following Type A and Type B methods of evaluation [24-26]. Each participating laboratory was requested to prepare an uncertainty budget at maximum pressure, considering all Type A and Type B uncertainty components.

\section{Reporting of Results and Data Analysis}

The values of measured pressure, acceleration of local gravity and reference temperature and the measurement uncertainty estimated at maximum pressure, reported by the participants are shown in Table 2. Measurement performance of the participants has been assessed on the basis of Error Normalized $\left(E_{n}\right)$ calculated for each measurement 
Evaluation of Laboratory Performance through Interlaboratory Comparison

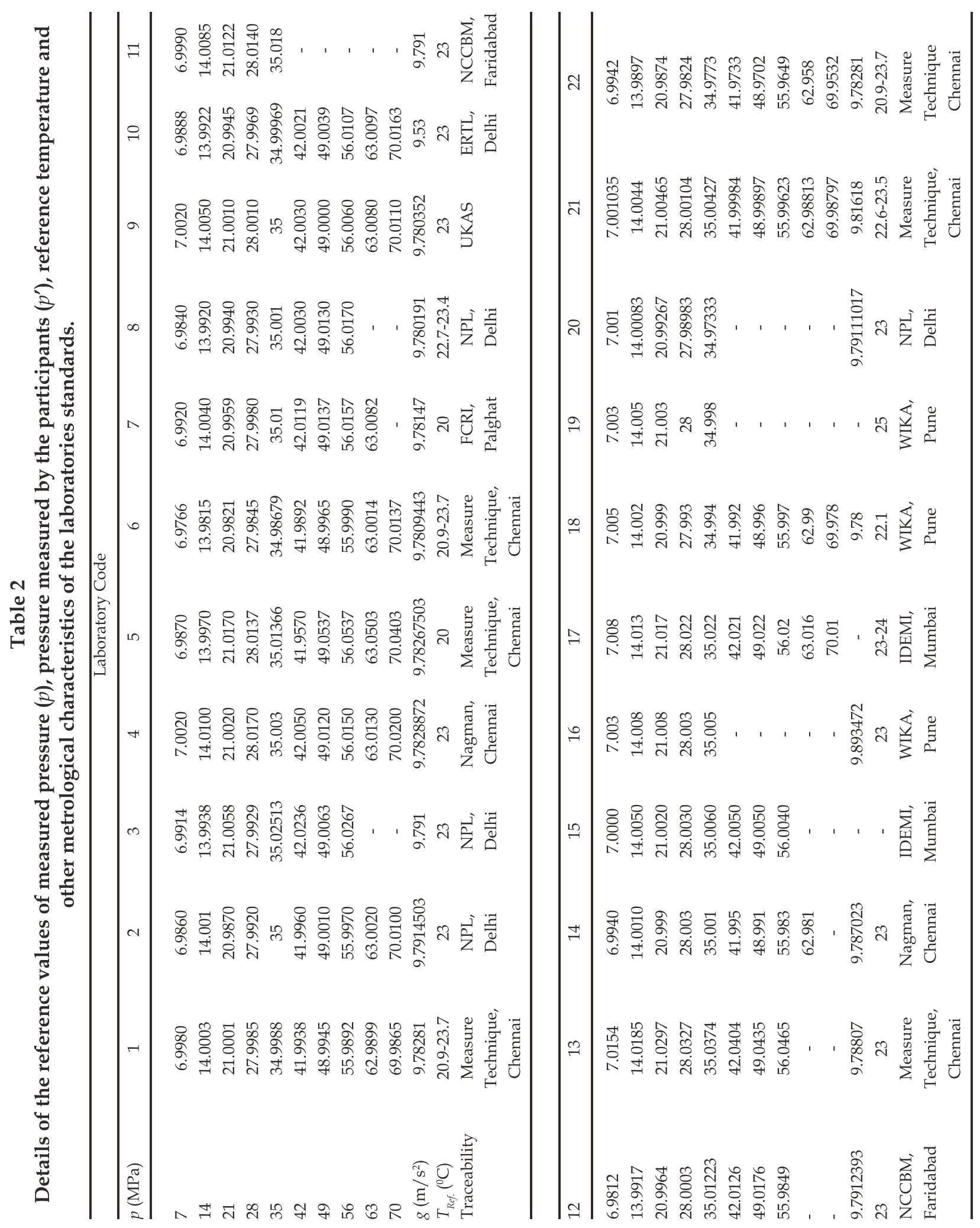


Sanjay Yadav and A.K. Bandyopadhyay
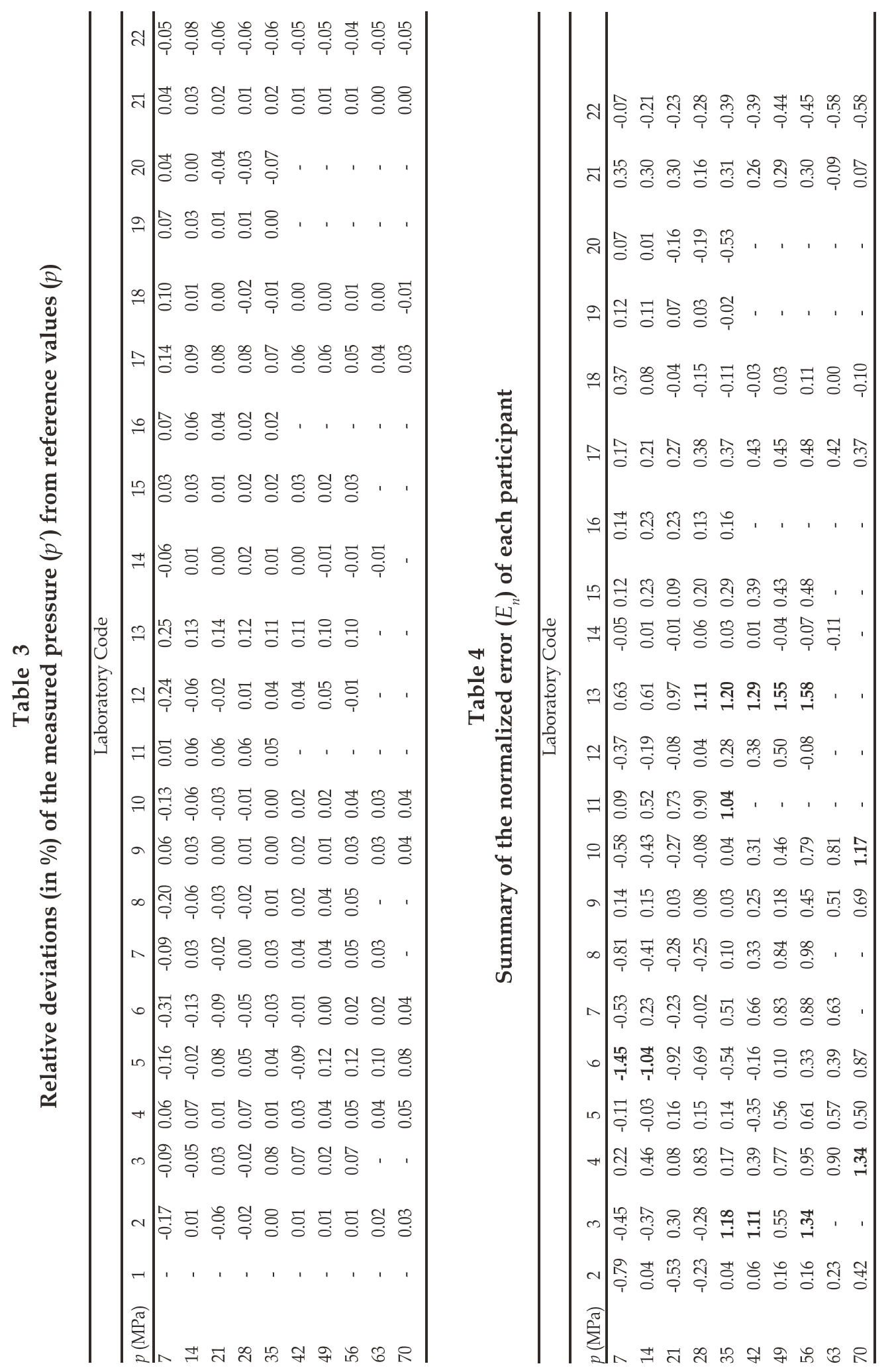
Evaluation of Laboratory Performance through Interlaboratory Comparison
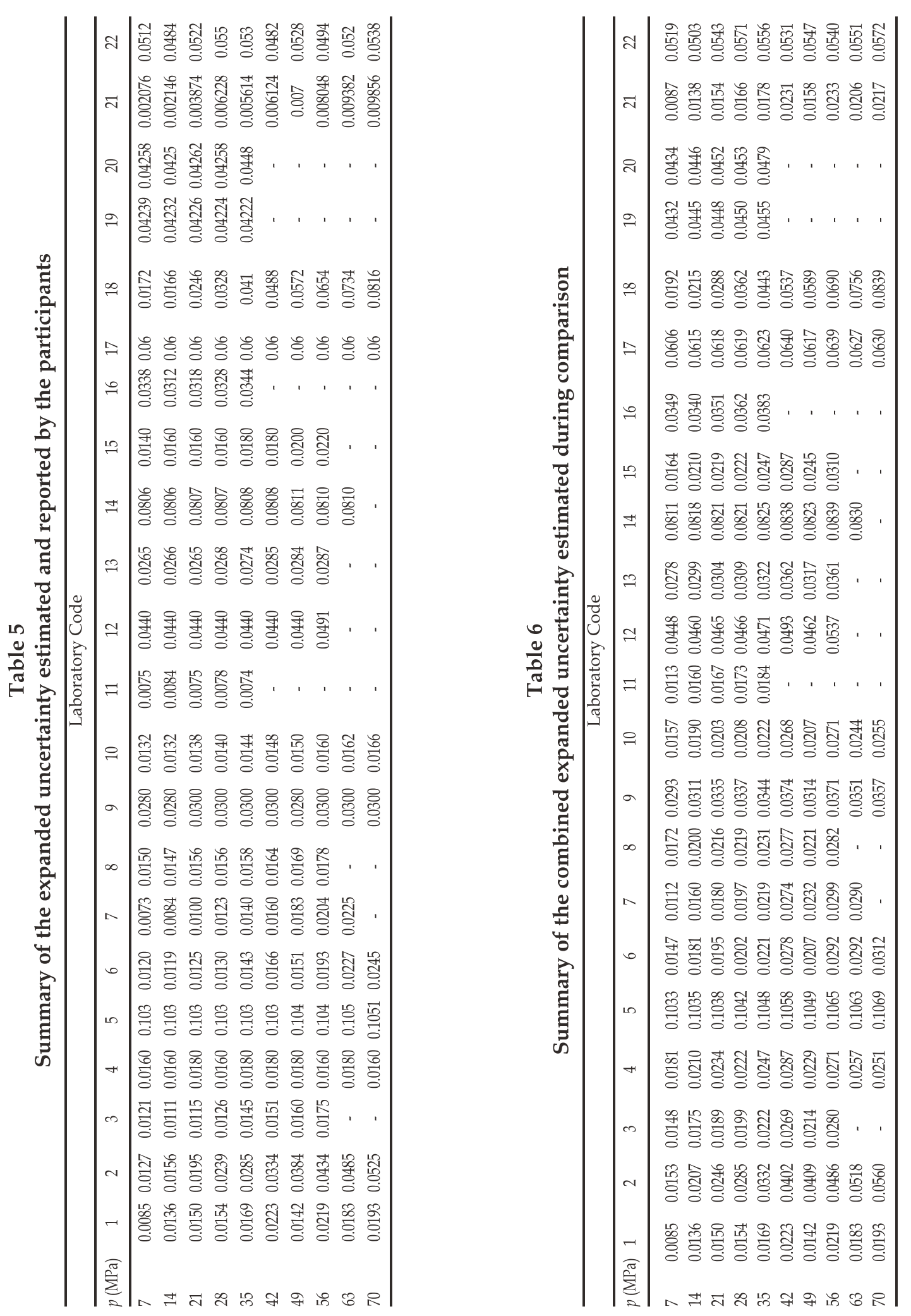
Sanjay Yadav and A.K. Bandyopadhyay

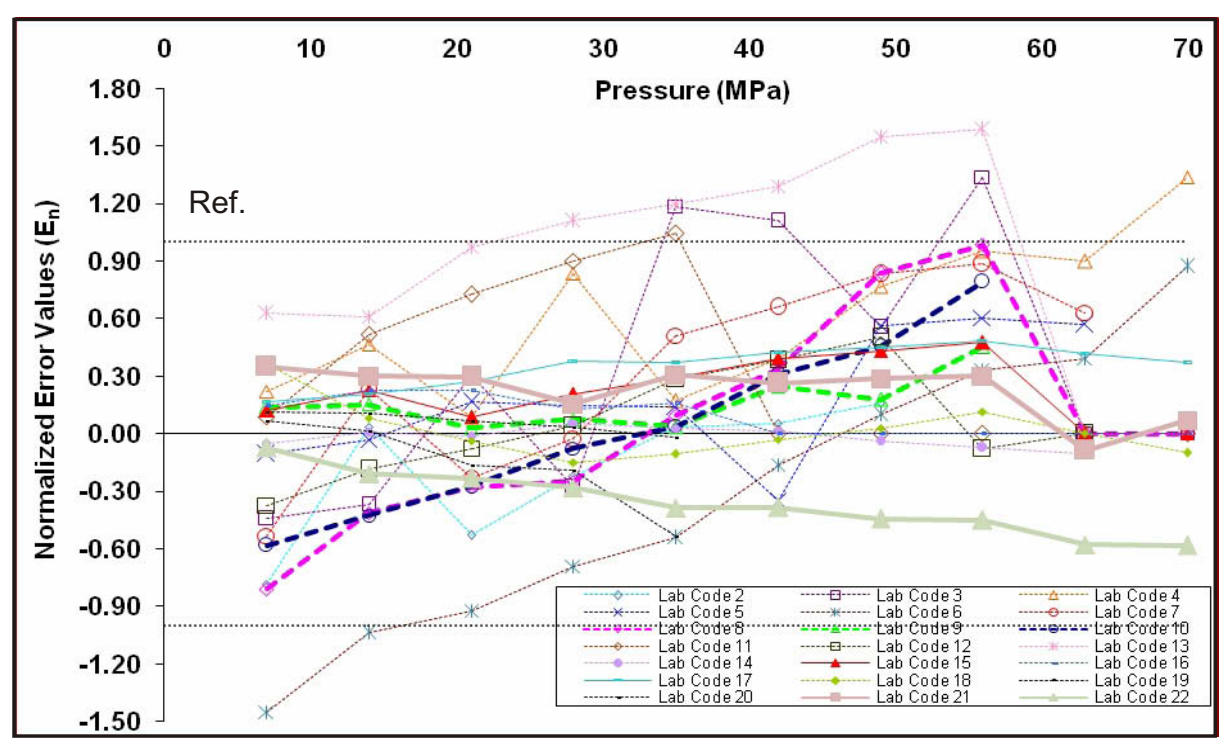

Fig. 4. The normalized error value $\left(E_{n}\right)$ as a function of measured pressure $\left(p^{\prime}\right)$ for each laboratory. The gap between two horizontal dotted lines shows the acceptable limit of the normalized error value

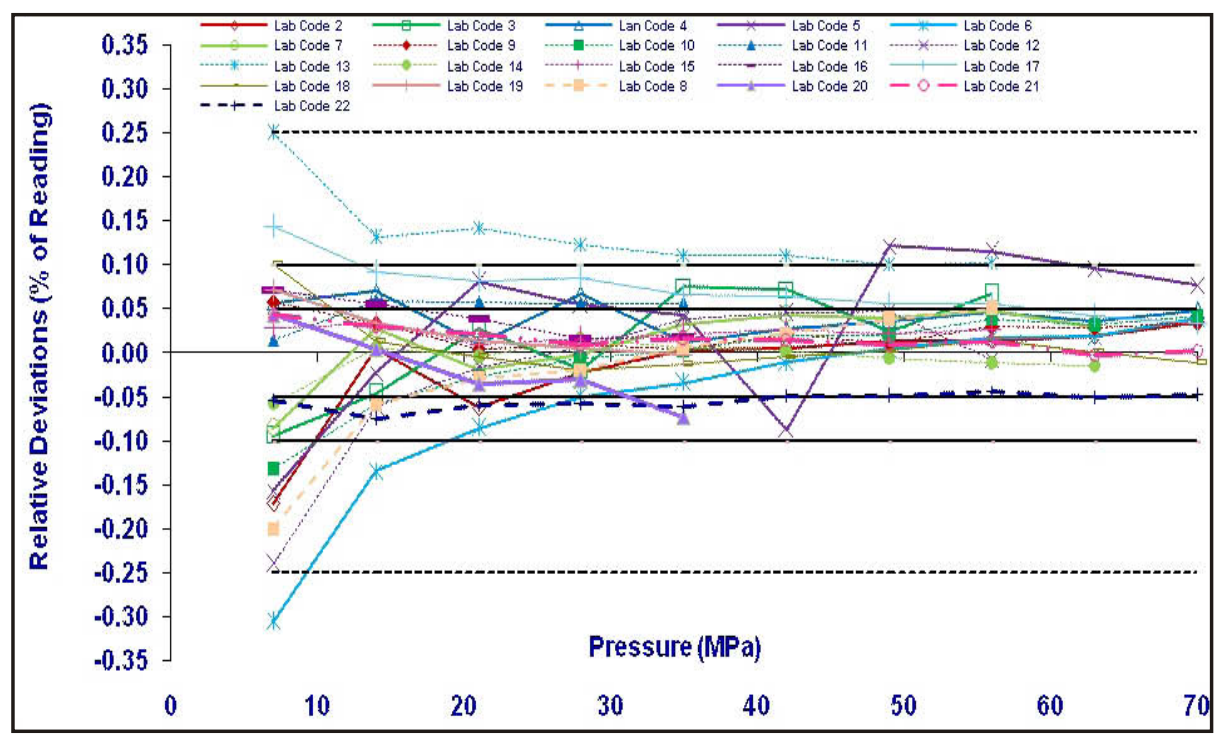

Fig. 5. Relative deviations of the measured pressure $\left(p^{\prime}\right)$ by each laboratory from the reference value

$(p)$. The gap between two horizontal dark black solid lines represents deviations falling within $\pm 0.05 \%$, dim black lines represents deviations falling within $\pm 0.1 \%$ and dotted lines represents deviations falling within $\pm 0.25 \%$

point. The $E_{n}$ values are estimated at each pressure as per guidelines in the literature $[7,15,24]$;

$$
E_{n}=\frac{p^{\prime}-p}{\sqrt{\left\{U\left(p^{\prime}\right)\right\}^{2}+\{U(p)\}^{2}}}
$$


where $p^{\prime}$ is the participant's measured pressure value, $p$ is the calculated reference value, $U\left(p^{\prime}\right)$ is the participant's claimed expanded uncertainty at a coverage factor $k=2$ and $U(p)$ is the expanded measurement uncertainty of the reference value at a coverage factor $k=2$. An $E_{n}$ value of less than 1 indicates agreement within the combined uncertainties for the results to be internationally acceptable. An $E_{n}$ number between-1 and +1 indicates an acceptable degree of compatibility between the laboratory's result and the reference value when the quoted uncertainties are taken into account. $E_{n}$ number $> \pm 1$ is unacceptable and requires immediate investigation and corrective action by the laboratory concerned.

\section{Results and Discussions}

The relative deviations of measured pressure, $p^{\prime}$, of each participant from reference value, $p$, are shown in Table 3 . The summaries of the normalized error values, $E_{n^{\prime}}$ estimated expanded measurement uncertainties, and the combined expanded measurement uncertainties are shown in Tables 4 to 6, respectively, for entire pressure scale of 7 to $70 \mathrm{MPa}$. The graphs plotted for the results for individual laboratories, are depicted as Figs. 5-6. In general, the performance of the laboratory is considered satisfactory if normalized error $E_{n}$ is $< \pm 1$. The plots shown in Figs. 4-5 reveal that there are total 178 measurement results. Measurement results of 15 laboratories (Code No. 2, 5, 7-9, 12 and 14-22) out of total 21 laboratories are well within acceptable limits of normalized error over the entire pressure range of 7 - $70 \mathrm{MPa}$. However, the measurement results of another 3 laboratories with Code No. 4, 10 and 11 are also quite good having $E_{n}$ values $> \pm 1$ only at one pressure point each. $E_{n}$ values of 165 measurement results out of total 178 are $< \pm 1$, which is $92.7 \%$. These results are acceptable. En values of remaining 3 laboratories referred by Code No. 3, 6 and 13 are $> \pm 1$ for 2 or more than 2 pressure points. The larger the absolute value of the $E_{n}$ number, the bigger the problem. An $E_{n}$ value greater than unity means that there is a significant bias in the laboratory's results and that the quoted value of its associated uncertainty does not adequately accommodate that bias, therefore further investigations are needed at the part of the laboratory.

The summary of the normalized error values $\left(E_{n}\right)$ as a function of applied pressure $\left(p^{\prime}\right)$ is depicted in Fig. 4. The relative deviations of measured pressure $\left(p^{\prime}\right)$ from reference values $(p)$ are shown in Fig. 5. The deviations lying within the uncertainty band of the reference laboratory is an indication of satisfactory results without any bias in the measurements. It is clearly evident from Fig. 5 that the relative deviations between laboratories values and reference values are well within the $0.05 \%$ for 123 measurement points, $0.1 \%$ for 162 measurement points and $0.25 \%$ for 177 measurement points. As reported earlier, this PT was organized for the laboratories having measurement uncertainty within $<0.05 \%$ to $>0.25 \%$ of full scale pressure, the relative deviations between laboratories values and reference values are well within $0.25 \%$ for all the pressure point except one pressure point of $7 \mathrm{MPa}$ for laboratory with Code No. 6. Further, the difference between the pressure values reported by the participating laboratories and the reference laboratory fall within the uncertainty band of reference laboratory at 121 measurement results i.e. $68.0 \%$, within their reported estimated expanded uncertainty band at 145 measurements results i.e. $81.5 \%$ and within the combined estimated expanded uncertainty band of this PT experiment at 165 measurement results (92.7 \%) (Fig. 4). Some of the laboratories, specially with Code No. 7, 11 and 21 have under estimated their measurement uncertainty at almost all the pressure points as shown in Table 5. Although, these laboratories have reported better measurement uncertainty than the reference laboratory, their $E_{n}$ values are found within acceptable limits except one pressure point of $35 \mathrm{MPa}$ for laboratory with Code No. 11. The main reasons for bias in the measurements may be due to errors in measuring instruments or in estimation / measurement of local acceleration of gravity, the error in applying the temperature and head corrections and the under estimation of measurement uncertainty. Laboratories would be able to rectify the problems by a review of their uncertainty calculations and other systematic affects as mentioned above.

All the participating laboratories were asked to submit the copy of the formal calibration certificate issued to the customer and traceability certificate of their standard. Almost all the laboratories submitted the copies of the formal calibration certificates of the digital pressure calibrator (artifact, in the present 
case) except 2 laboratories with Code Nos. 11 and 12 . The traceability certificates were also received from 18 laboratories. Laboratories with code numbers 2, 11 and 14 did not submit such formal certificates. Certificates thus received were examined and found adequate except that there is little uniformity in the calibration certificates issued by the participants, specially in reporting the measurement results. There are few typo errors in some of the certificates, for example, capital ' $K$ ' for coverage factor ' $k$ ' (laboratories with Code numbers 5, 15 and 16; also traceability certificate of Code No. 17), in pressure unit as ' $\mathrm{kg} / \mathrm{cm}^{\prime}$ in place of ' $\mathrm{kg} / \mathrm{cm}^{2}$ (laboratory with Code No. 3), used 'fro' in place of 'from' (laboratory with Code No. 4), in pressure unit as ' $\mathrm{KPa}$ ' in place of ' $\mathrm{kPa}^{\prime}$ (laboratory with Code No. 6) and wrong reporting of unit of ' $\mathrm{g}^{\prime}$ ' as ' $9.7865244 \mathrm{~cm} / \mathrm{s}^{2 \prime}$ in place of ' $9.7865244 \mathrm{~m} / \mathrm{s}^{2 \prime}$ (traceability certificate of the laboratory with Code No. 16). Although, these are minor errors but should have been avoided.

As mentioned earlier, $E_{n}$ numbers greater than unity require investigations and corrective action by the participating laboratory. The laboratory's management needs to ensure that the problem is rectified and procedures are put in place to prevent a recurrence. Laboratories with Code No. 3, 6 and 13 were asked to review the results and take appropriate corrective actions. All these laboratories were asked to improve their calibration facilities and to modify the measurement method and to estimate the measurement uncertainties properly. Laboratories with Code No. 4, 10 and 11 may also be requested to review their results at their respective unacceptable measurement points (one each). During the followup process, these three laboratories have addressed the reasons for their results being outside the range, and they were advised to take part in at least one interlaboratory comparison with another laboratory having better measurement capabilities.

\section{Conclusion}

This interlaboratory comparison programme (proficiency testing) is carried out in the pressure range 7 - $70 \mathrm{MPa}$ using pressure digital pressure calibrator as an artifact. Total number of 21laboratories participated in this programme. The comparison was performed at 10 equally spaced pressure points selected throughout the entire pressure range. The proficiency testing concludes that out of the total 178 measurement results reported here in this report, $165(92.7 \%)$ are in agreement with the reference laboratory. The $E_{n}$ values of 15 laboratories are within acceptable limits through out the entire pressure scale. However, the $E_{n}$ values of 3 other laboratories are also quite acceptable except only at one pressure point each. The $E_{n}$ values of the remaining 3 laboratories are found beyond the acceptable limit for 2 or more than 2 pressure points. The difference between laboratories values and reference values of 121 measurement points $(68.0 \%)$ are well within the uncertainty bands of the reference values. Total 145 measurements results i.e. $81.5 \%$ fall within their reported expanded uncertainty band. However, $92.7 \%$ measurement results are found well within combined estimated expanded measurement uncertainty band. Since some of the laboratories have under estimated their measurement uncertainties, $7.3 \%$ measurement results are found out of the combined uncertainty band during this comparison. The measurement process used for the present PT is quite good and may be used by other PT organizers for the assessment of measurement capabilities of their participating laboratories. The whole PT experiment was completed without any problem and technical issue. Since this is the first proficiency test experience for most of the participating laboratories, the results are considered to be very good.

\section{Acknowledgements}

We are grateful to Dr. Vikram Kumar, Director, National Physical Laboratory, New Delhi and Dr. Hari Gopal, Director, National Accreditation Board for Testing \& Calibration Laboratories, New Delhi for their support and encouragement. We are also thankful to our colleagues Dr. P.C. Kothari, Dr. K.K. Jain, Mr. A.K. Saxena and Dr. Naveen Garg for their constant co-operation and time to time suggestions and discussions. Thanks to the seventeen accredited laboratories for completing the PT on time. We would also like to acknowledge the help of the secretariat of NABL for their administrative help and our colleagues Mr. B.V. Kumaraswamy for critically reviewing this report, Mr. V.K. Gupta and Mr. Om Prakash for assisting in experiments.

\section{References}

[1] "High Pressure Measurement Techniques", edited by Peggs G.N., Published by Applied 
Evaluation of Laboratory Performance through Interlaboratory Comparison

Science Publishers, London, U.K. (1983).

[2] R. S. Dadson, S. L. Lewis and G. N. Peggs, "The Pressure Balance: Theory and Practice", Published by Her Majesty's Stationary Office, London, (1982).

[3] F. Pavese and G. Molinar, "Modern Gas Based Temperature and Pressure Measurements", Plenum Press, New York, (1992).

[4] Sanjay Yadav, Ravinder Agarwal, A.K. Bandyopadhyay, D.R. Sharma and A.C. Gupta, "Modern Instrumentation Techniques in Pressure Metrology under Static Conditions", Mapan- Journal of Metrology Society India, 18 (2003) 57-82.

[5] “General Requirements for the Competence of Testing and Calibration Laboratories", ISO / IEC 17025: (1999).

[6] "Procedures for Establishing and Maintaining the APLAC Mutual Recognition Arrangement Amongst Accreditation Bodies", APLAC MRA001, 13 (2007).

[7] "Proficiency Testing by Interlaboratory Comparison: Part - 1: Development and Operation of Proficiency Testing Schemes. Part - 2: Selection and use of Proficiency Testing Schemes by Laboratory Accreditation Bodies", ISO / IEC 43 (1997).

[8] Sanjay Yadav and A.K. Bandyopadhyay, "Proficiency Testing Program Under NABL in the Pressure Range 7-70 MPa Using a Dead Weight Tester", Med. J. Meas. Contrl. (UK), 1 (2005) 138-151.

[9] Sanjay Yadav and A.K. Bandyopadhyay, "Interlaboratory Comparison in the Pressure Range up to $70 \mathrm{MPa}$ using Dead Weight Tester as an Artifact (NABL -Pressure -PT004)", Technical Protocol (2007).

[10] Sanjay Yadav and A.K. Bandyopadhyay, "Proficiency Testing (PT) Program Under NABL in the Pressure Range 7 - $70 \mathrm{MPa}$ ", Metrology and Measurement Systems, Poland, XII (2005) 323-340.

[11] Sanjay Yadav and A.K. Bandyopadhyay, "Interlaboratory Comparison in the Pressure Range 7 - $70 \mathrm{MPa}$ Using Digital Pressure Calibrator", Mapan- Journal of Metrology
Society of India, 20 (2005) 2 97-310.

[12] Sanjay Yadav, V.K. Gupta, Om Prakash and A.K. Bandyopadhyay, "Evaluation of Interlaboratory Performance Through Proficiency Testing using Pressure Dial Gauge in the Hydraulic Pressure Measurement up to $70 \mathrm{MPa}$ ", Mapan- Journal of Metrology Society India, 23 (2008) 79-99.

[13] Sanjay Yadav, V.K. Gupta, Om Prakash and A.K. Bandyopadhyay, "Proficiency Testing Through Interlaboratory Comparison in the Pressure Range 7-70 MPa Using Pressure Dial Gauge as an Artifact", J. Sci. and Indusl. Res., 64 (2005) 722-740.

[14] Sanjay Yadav and A.K. Bandyopadhyay, "Assessment of Laboratory Performance in External Proficiency Testing in the Pressure Range up to $60 \mathrm{MPa}$ " Measure, USA, 4 (2009) 42-51.

[15] "Guidelines for Proficiency Testing Program for Testing and Calibration Laboratories", NABL Doc. 162 (2001).

[16] Sanjay Yadav, A.K. Bandyopadhyay and A.C. Gupta, "Characterisation of National Hydraulic Pressure Standards in the Pressure Ranges up to $100 \mathrm{MPa}, 200 \mathrm{MPa}$ and 500 MPa", Callab: The International J. Metrology, USA, (2003) 28-35.

[17] Sanjay Yadav, A.K. Bandyopadhyay, N. Dilawar and A.C. Gupta, "Re-establishment of Measurement Uncertainty in Pressure Measurement Through In-house Laboratory Intercomparison of National Hydraulic Pressure Standards up to $500 \mathrm{MPa}$ ", Mapan Journal of Metrology Society of India, Suppl. 1(2001) 170-177.

[18] Sanjay Yadav, A.K. Bandyopadhyay, N. Dilawar and A.C. Gupta, "Intercomparison of National Hydraulic Pressure Standards up to $500 \mathrm{MPa}$ ", Measurement + Control, UK, 35 (2002) 47-51.

[19] A. K. Bandyopadhyay, Sanjay Yadav and Nita Dilawar, "Current Status of Pressure Standards at NPLI and Our Experiences with the Key Comparison Data Base (KCDB)" Mapan- Journal of Metrology Society India, 21 (2006) 127-145. 
[20] R G Driver, D A Olson, Sanjay Yadav, A K Bandyopadhyay, "Final Report on APMP. SIM.M.P-K7: Bilateral Comparison Between NIST (USA) and NPLI (India) in the Hydraulic Pressure Region $40 \mathrm{MPa}$ to $200 \mathrm{MPa}^{\prime \prime}$, Metrologia Tech. Suppl., France, 43 (2006) 1-15.

[21] Sanjay Yadav, D. Arun Vijayakumar and A. C. Gupta, "Computer Software for Calibration of Industrial and Master Simple / Reentrant Type Piston Gauges", Mapan- Journal of Metrology Society India, 12 (1997) 101-104.

[22] D. Arun Vijayakumar, Sanjay Yadav and A.C. Gupta, "Quality of Measurements: a Software for Estimation of Measurement Uncertainty", Presented during National Symposium on Electronics in Societal Mission, New Delhi, March (1997) 29-30.
[23] Sanjay Yadav, V.K. Gupta, Om Prakash and A. K. Bandyopadhyay, "Evaluation of Associated Uncertainties in Calibration of Direct Pressure Indicating Electromechanical Devices", Measurement Science Review, 5 (2005) 104-114.

[24] H.S. Nielsen, “Determining Consensus Values in Interlaboratory Comparisons and Proficiency Testing" Proceedings NSCL Conference, August 17-18, Tampa, Florida, USA, (2003) 1-16.

[25] "Guide to the Expression of Uncertainty in Measurement", ISO Document - ISO/TAG/ WG 3: 1995 (E).

[26] "Guidelines for Estimation and Expression of Uncertainty in Measurement", NABL Doc. (2000) 141. 